\title{
Élèves associés
}

Témoignage à l'école allemande de Paris

Associating pupils - the example of the German School in Paris

Alumnos asociados. Testimonio en el colegio alemán de París

\section{Roger-François Gauthier}

\section{OpenEdition}

\section{Journals}

Édition électronique

URL : https://journals.openedition.org/ries/1865

DOI : 10.4000/ries. 1865

ISSN : 2261-4265

\section{Éditeur}

France Education international

Édition imprimée

Date de publication : 1 avril 2002

Pagination : 65-73

ISBN : 2-84520-553-8

ISSN : $1254-4590$

Référence électronique

Roger-François Gauthier, "Élèves associés », Revue internationale d'éducation de Sèvres [En ligne], 29 avril 2002, mis en ligne le 25 novembre 2011, consulté le 06 juillet 2021. URL : http:// journals.openedition.org/ries/1865; DOI : https://doi.org/10.4000/ries.1865 


\section{Élèves associés}

\section{Témoignage à l'école allemande de Paris}

\section{Roger-François Gauthier}

À l'école allemande de Paris, la confrontation collective à une situation problème, constamment préférée à un exposé magistral du savoir, suscite d'autant mieux la motivation des élèves qu'ils sont associés à certains choix pédagogiques.

Il suffit de passer le pont, non celui de Kehl, mais celui de Saint-Cloud, et, comme dans la chanson, c'est tout de suite sinon l'aventure, au moins l'étonnement, pour qui s'intéresse à la matière pédagogique. Partie intégrante du système éducatif allemand, et donc rattachée administrativement à l'un des Länder de façon tournante, l'école allemande de Paris, de la maternelle à l'Abitur ${ }^{1}$ entraîne des élèves et rassemble des professeurs qui ont assez souvent eu l'occasion de se frotter au collège et au lycée français, ce qui leur permet, plus facilement qu'à d'autres, quand ils « comparent », avec la force du vécu, de pointer des différences de fond.

La prudence méthodologique impose bien sûr de se demander, à propos de ce qui peut être dit et observé dans une telle école, si les modalités pédagogiques et les moyens disponibles sont conformes à ce qui se passerait dans un établissement d'Allemagne et si le recrutement socio-culturel spécifique des élèves ne modifie pas les conditions d'enseignement de telle manière que, là encore, l'observation ne serait pas significative. C'est en faisant sienne cette prudence que l'auteur des entretiens avec les responsables de l'établissement, avec des professeurs et avec des élèves dont on trouve ici la relation s'est attaché à vérifier chaque fois que ce qui était constaté à l'école allemande de Paris ne valait pas qu'au bord de la Seine.

Passons le pont et d'abord, s'agissant de la forme des entretiens, remarquons que, pour répondre à une demande d'un responsable d'un système éducatif étranger qui frappait à la porte, comme ce fut le cas du rédacteur de cet article, pour enquêter, disait-il, sur le "travail des élèves », la direction de l'établissement lui proposa certes de rencontrer des professeurs, donc des adultes et des prescripteurs, mais surtout de passer l'essentiel du temps en audition avec... des élèves, avec, par exemple, une classe entière (des élèves de

1. C'est-à-dire l'« équivalent » du baccalauréat d'enseignement général. On tiendra ici pour connus du lecteur certains traits essentiels du système éducatif allemand, qu'il s'agisse de l'orientation très précoce, dans la plupart des länder, dès l'âge de dix ans, des élèves entre les trois voies, Gymnasium, Realschule et Hauptschule (à noter au passage que ces troies voies coexistent à l'école de Paris) ou des spécificités du temps scolaire allemand, sans cacher toutefois les interférences de ce thème avec les activités de l'élève, plus disponible pour le travail autonome et devant d'autant mieux « rentabiliser » l'horaire de cours que celui-ci est plus restreint. 
terminale) auxquels la direction de l'établissement avait spontanément ouvert la possibilité de dire ce qu'ils voulaient sur le sujet de leur travail, pendant l'horaire habituel d'une discipline, au visiteur étranger.

Notons encore que les professeurs de ces élèves dans plusieurs disciplines assistaient à la classe ainsi «banalisée ». Ils «n'occupaient pas le terrain » de la parole, aucun n'a jamais coupé celle d'un élève et ils intervenaient au même niveau qu'eux.

Une fois franchi le fleuve, Seine ou Rhin, ce qui étonne nous conduit à considérer successivement :

- la gestion de la classe, pour savoir quelle place y tient l'activité de l'élève ;

- le contexte qui permet ce qui aura été préalablement énoncé : un rapport peut-être différent de l'élève à son maître, aux programmes d'enseignement, aux examens et à ses propres outils de travail ;

- la conscience étonnamment exprimée par les élèves de leur propre participation à certains choix pédagogiques.

\section{L'ACTIVITÉ DE L’ÉLÈVE AU CENTRE DE LA GESTION DE LA CLASSE}

Les élèves qui avaient fréquenté le système français insistaient sur le fait que, selon eux, dans la classe française, le sujet traité apparaît d'emblée comme «abstrait», partie d'un programme "académique», censé connu du seul professeur qui dispense un "savoir"; l'élève, toujours selon leurs "impressions ", passe l'essentiel de son temps à écrire tandis qu'ils ont conscience, dans le système allemand, de passer l'essentiel du leur à parler.

Pour les élèves allemands, auxquels nous laissons ici la parole au style indirect, la classe est constamment vécue comme un lieu de recherche des solutions à un problème certes proposé par l'enseignant, mais dont l'enjeu leur apparaît clairement; cette recherche est totalement collective, associe tous les élèves qui se sentent tenus par le groupe de participer à l'effort collectif, un peu de la même façon qu'on attend sa contribution de chaque joueur d'une équipe sportive.

Les élèves expriment qu'ils ont le sentiment de peu écrire, seulement à certains moments de la séquence, et essentiellement pour noter les points sur lesquels a pu se constituer l'accord et que le professeur a consignés au tableau.

L'oral est naturellement prépondérant ; le désordre qu'il crée temporairement est réputé normal ; la spontanéité de l'expression est importante ${ }^{2}$; la régulation appartient au maître dans la forme, au groupe dans le fond, qui veille

2. « Interrompre » n’est pas mal vu ici ! » affirmait un élève qui avait fréquenté des établissements français. 
à ce que la question avance. La tension des " heures » de cours, qui ne durent que 45 minutes, est décrite comme collectivement plus forte, et individuellement moins stressante que dans la classe française. Les élèves expriment que c'est pour eux au fond le groupe qui est appelé à penser par lui-même, professeur inclus, l'apprentissage de l'autonomie s'effectuant au sein du groupe. L'idée est que chaque individu trouve dans le cadre, décrit comme sécurisant, du travail du groupe, un entraînement à sa propre autonomie ${ }^{3}$.

Du côté des professeurs, le plus immédiatement étonnant est sans doute, l'unisson pédagogique des différentes disciplines pour définir, comme leurs élèves, la classe comme ce lieu de travail, et de travail commun aux enseignants et aux élèves autour d'un sujet qui fait problème. Les professeurs ont confirmé ce que leurs élèves avaient annoncé à leur manière, à savoir que la gestion de la classe par la recherche collective de solutions à un problème était le mode habituel quels que soient les niveaux et les disciplines et qu'ils avaient comme leurs élèves leurs repères dans ce type de fonctionnement parce qu'il était commun à l'ensemble des disciplines.

Le témoin français de ces pratiques, l'auditeur français de ces témoignages objectera que ce qui est évoqué là n'est que la conduite d'une classe vivante telle qu'elle est, Dieu merci, pratiquée par beaucoup de professeurs français : c'est là précisément qu'il convient d'approfondir l'analyse pour voir en quoi certains aspects de l'environnement pédagogique allemand peuvent favoriser non pas l'émergence de ce type de conduite de classe, mais sa généralisation.

La question devient donc : "Pour quels motifs la classe allemande parviendrait-elle plus souvent à être cela et, partant, à motiver les élèves et à mettre à leur portée un rapport au savoir plus autonome? » Nous ne retiendrons que les éléments du contexte qui concernent la pédagogie, et non ce qui relève de choix politiques historiques de l'école des deux pays.

\section{UN AUTRE RAPPORT À L'ENSEIGNANT, AU SAVOIR, À L'EXAMEN ET À L'OUTIL}

Comparer des situations étrangères en matière éducative pose chaque fois la question de l'importance à accorder au «contexte » : à l'évidence l'ignorer conduit à des contresens et à des impasses pour l'action, mais le connaître peut conduire tout aussi vite à la paralysie Chacun recevra au moins

3. Tout est bien sûr dans tout et réciproquement : la crainte, que le lecteur français ressent, peut-être, que l'importance donnée au groupe et à l'oral ne privilégie les élèves d'un certain type trouve sa réponse dans la partition habituelle du secondaire allemand en trois voies ; à l'intérieur de chacune des voies, naturellement, l'hétérogénéité, quant aux types d'élèves, et aussi naturellement quant à leurs origines, est limitée. La différence fondamentale entre les contextes politiques de l'éducation dans les deux pays trace ici les limites, réelles mais dont il appartient à chacun d'estimer si elles sont franchissables, de toute transférabilité en matière pédagogique. 
ces éléments d'analyse comme des interpellations dont il lui appartiendra de chercher s'il peut, là où il est, du citoyen à l'enseignant ou au ministre, en tirer quelque chose :

\section{La relation entre les élèves et les enseignants}

La relation entre les élèves et les enseignants est plus une relation de travail que d'autorité. C'est sans doute un des points les plus connus, sur lequel nous ne nous attarderons pas. On notera donc de façon à dessein impressionniste les points suivants :

- plusieurs fois des enseignants ont interrompu l'échange parce que sonnait l'heure de la «récréation » et qu'ils étaient de garde pour aller dans la cour. Ils expliquaient spontanément qu'il s'agissait moins de "surveillance» que d'un moment privilégié pour que les élèves puissent leur parler ;

- les enseignants acceptent explicitement d'être définis par leurs propres élèves, et en leur présence, comme des «modérateurs " dans l'activité de la classe : le professeur s'affiche comme maître d'une relation pédagogique avant de s'afficher comme le dépositaire et transmetteur d'un savoir; devant la situation-problème qu'il propose, peu importe qu'il «sache " ou qu'il «sache faire », ce qu'on attend de lui, c'est qu'il « sache faire faire », artisan d'un travail collectif au sein duquel il joue le jeu en acceptant de courir des risques contrôlés mais réels ;

- les enseignants affirment s'adresser à des "enfants» plus qu'à des «élèves ». Cette précision ne manque pas d'étonner un auditeur français, pour lequel précisément l'école «institue » l'élève. Si le mot n'a pas la faveur des professeurs allemands, c'est qu'il désigne pour eux un être abstrait, dessiné par une institution sur le mode du futur ("si l'éducation t'“élève”, c'est pour que plus tard tu...»), tandis qu' "enfant » se réfère plutôt à la personne qu'est déjà cet élève, déjà embarquée dans cette vie, et confrontée aux problèmes ordinaires des humains ${ }^{4}$. Si ce n'est pas le lieu ici de se pencher sur le contenu éducatif des relations que les enseignants allemands entretiennent avec leurs élèves et les familles de ces derniers, on retiendra que l'exemple suivant a été cité pour valoir autre chose qu'une anecdote : des élèves nous ont spontanément dit avec quelle surprise ils ont entendu le ministre français de l'éducation nationale demander aux enseignants du pays d'aborder avec leurs élèves le drame du World Trade Center : il était pour eux évident que leur école allait les "faire travailler»sur un tel sujet, parce que c'était leur vie qui l'impliquait ${ }^{5}$.

\footnotetext{
4. La thèse implicite est claire : l'« élève » français est en fait « infantilisé » alors même que l'école refuse de faire de lui un " enfant ", tandis qu'en lui reconnaissant ce statut, la classe allemande le rapproche du statut de la " personne » et lui permet ainsi de mieux grandir. Le moins que l'on puisse dire est que cela mérite discussion !

5. Le temps a manqué pour savoir en quoi a précisément consisté ce travail, mais il paraissait avoir été conduit en réponse aux questionnements des élèves sur les évènements.
} 


\section{Un rapport au savoir entre synthèses et approfondissements actifs}

L'objection la plus souvent rencontrée en France par ceux qui prônent le développement des activités des élèves est celle du temps requis : on nie rarement que l'apprentissage puisse être de meilleure qualité quand l'élève a une part plus active dans l'invention des solutions à des problèmes ressemblant eux-mêmes plus à des études de cas qu'à des constructions abstraites sentant la craie et le tableau noir, mais on regrette qu'il faille consacrer à de telles séances un temps dont on ne dispose pas. On ne pourra pas, dans ces conditions, "finir le programme ». Ne prenons pas ici l'argument à la légère car l'étude des programmes d'enseignement est effectivement une des obligations de base de l'école et peutêtre sa raison d'être : il y a simplement cette difficulté qu'un programme donne rarement le détail de l'approfondissement qu'il convient d'atteindre sur chacun des sujets et que, en tous les cas, c'est bien face à ce caractère synthétique du libellé d'un programme que le professeur doit déployer une stratégie.

Dans la situation allemande, il est explicitement reconnu par la réglementation en vigueur que tout ne sera pas traité avec le même approfondissement, ni l'objet d'autant d'attention de la part des élèves, mais on dit cela positivement : tout sera vu «dans les grandes lignes». La gestion des programmes d'enseignement a droit aux changements de rythme, à une polyrythmie explicite vis-à-vis des élèves.

Dans le cadre d'un programme qui ressemble formellement à un programme français, la réglementation impose de tout étudier, mais les professeurs doivent distinguer ce qui sera vu « dans les grandes lignes", et ce qui sera approfondi : un choix est à faire, qui sera la plupart du temps le lieu d'une négociation entre le professeur et ses élèves, des sujets qui donneront précisément lieu aux séances collectives de travail sur des situations faisant problème.

Du même coup, comme on accepte d'aller plus vite sur beaucoup de choses, on peut se permettre de creuser beaucoup plus certaines autres : on peut donc se demander ce qui est préférable, de l'ambition apparemment « encyclopédique » de la gestion française des programmes, ou de l'attitude de survol délibérément affiché comme tel avec des plongées en eau profonde à quelques étapes négociées, selon ce mode d'" approfondissement inégal » caractéristique de la gestion allemande. La latitude ainsi donnée aux classes bénéficie en tous les cas, selon les disciplines, à des travaux dont le moins qu'on puisse dire est qu'ils ne paraissent pas relever du laxisme : en langues étrangères, par exemple, la lecture de plusieurs œuvres complètes, plongée sans masque, en apnée dans un auteur ${ }^{6}$, est le mode normal de travailler.

6. En anglais, dans une terminale pas spécifiquement littéraire, puisque cette partition n'existe pas, Macbeth est dans l'ordre courant de ce qu'on lit, en œuvre intégrale. 
Comme nous savons que la liberté de mouvement des classes est souvent bridée par la pratique et la réglementation des examens, nous avons à nous demander dans quelle mesure les pratiques d'évaluation sont conformes à l'esprit de cette pédagogie.

\section{Le rôle des pratiques d'évaluation}

Les pratiques d'évaluation, en responsabilisant les classes, contribuent de leur côté à valoriser le travail des élèves. Connu par l'expérience directe que certains élèves et professeurs en avaient ou par ce que des amis leur en avaient rapporté, le fonctionnement des examens de l'enseignement secondaire français était critiqué de façon intéressante : rapporté au système allemand d'examens, le baccalauréat par exemple était décrit notamment comme effrayant par l'étendue des programmes qu'il impose de couvrir, comme aléatoire en raison de la diversité des questions qui peuvent "tomber » et comme faisant une part prépondérante à l'écrit au détriment d'un oral ramené à un tête-à-tête avec un unique examinateur.

L'Abitur permet de comprendre en quoi l'examen induit en amont un autre type de "travail » pour les élèves; on peut par exemple noter :

- que les sujets des épreuves écrites ${ }^{7}$ sont propres à l'établissement, choisis par une autorité extérieure parmi ceux que chaque professeur a proposés en fonction des questions qu'il a particulièrement traitées avec ses élèves et à propos desquelles il a dit à l'inspecteur comment il les a traitées ;

- que les épreuves orales ne mettent jamais un examinateur unique en face d'un candidat, et que les examinateurs de l'oral ont à justifier au jury par écrit leurs appréciations;

- que l'examen comprend une part importante de ce que permet mal de désigner l'expression française de contrôle continu, mais qui, tout en restant une évaluation décrite par les uns et les autres comme rigoureuse, valorise aux yeux de l'élève le travail qu'il fournit durant les deux dernières années de sa scolarité.

L'avocat du diable, s'il y avait procès, fustigerait une scolarité qui place ainsi l'élève dans une préoccupation permanente de l'examen, puisque tout paraît «compter»». La réalité est plus intéressante : l'implication de l'élève dans le travail collectif de la classe est prise en compte pour l'examen, mais en tant que mesure d'une activité et non d'un « niveau »; quant aux évaluations de cours d'année "qui comptent», ou bien "sommatives», dirions-nous en d'autres termes, elles sont au nombre de deux ou trois chaque semestre, avec des dates et des programmes arrêtés longtemps à l'avance. Ces «compositions»

7. Pour ce motif ils ne sont pas « nationaux » stricto sensu, mais ils sont arrêtés par un inspecteur qui vérifie leur adéquation à des programmes qui, eux, sont nationaux. 
permettent d'autant mieux un travail véritable qu'elles bénéficient d'un horaire conséquent ( 4 heures par exemple en allemand en classe terminale).

Le rapport entre le travail des élèves et les évaluations en cours d'année ou à l'occasion d'un examen paraît ainsi échapper à certaines contradictions bien connues et résistantes dans d'autres systèmes : l'activité des élèves en cours d'année est par elle-même notée et valorisée pour l'obtention du diplôme, et le contrôle terminal, ne "prenant pas les élèves en traître ", valorise une seconde fois leur travail de l'année puisqu'il porte sans surprise sur ce que l'élève a effectivement pu travailler en profondeur.

À noter aussi que si la contradiction entre la richesse du travail des élèves pendant l'année et la passation d'épreuves d'examens est en partie levée par ces pratiques, il est un autre motif pour lequel leurs activités ne se réduisent pas à la perspective de l'examen : pendant leur temps libre, les élèves se voient proposer nombre d'activités facultatives qui ne "rapportent " en aucun cas de points pour l'obtention de l'examen, mais dont il est significatif de voir que les élèves, outre l'intérêt de ces pratiques pour leur enrichissement personnel, pensent qu'elles pourront témoigner de leur personnalité quiconque lira leur dossier scolaire au-delà de l'examen (lors d'une " embauche », selon les lycéens rencontrés, ou pour candidater à une poursuite d'études, ...).

\section{L'importance de l'outillage de l'élève}

La question des outils nécessaires au travail des élèves est apparue dans les propos des élèves comme de leurs maîtres avec une importance surprenante, et sans même qu'il soit particulièrement question des technologies les plus récentes. Les élèves ont insisté sur quatre types d'outils, dont la seule évocation est significative :

- certains outils sont indispensables à la continuité des études et servent de repère d'une année sur l'autre : il est par exemple prescrit de garder ses manuels d'histoire des années antérieures et un professeur d'une année successive peut demander de s'y référer; il en va de même dans plusieurs disciplines (autre exemple : les manuels et grammaires d'allemand et de langues vivantes étrangères) où le manuel de classe est plus une référence qu'un objet éphémère à revendre ou à rendre à un organisme de prêt ;

- d'autres outils, sans être en propre des outils scolaires, sont clairement valorisés dans les classes et il n'existerait pas d'obstacle à prescrire aux familles l'acquisition de telle encyclopédie, ou de tel atlas, sans parler des dictionnaires ; la classe étant conçue comme le lieu d'un travail, l'élève a besoin, en dehors d'appuis documentaires variés sur chaque sujet, de cette trousse de base dont l'école n'hésite pas à fixer le contenu ;

- les ressources documentaires au sens plus large qui furent citées étaient de celles qu'il est d'usage de trouver dans les centres de documentation 
et d'information des établissements d'enseignement, l'enthousiasme des élèves à évoquer l'importance cruciale de ces ressources pour leur travail quotidien étant seul à relever ;

- furent enfin cités comme indispensables, notamment pour soulager la mémoire et dégager ainsi de l'énergie pour des tâches plus ambitieuses tous les outils autorisés aux examens et épreuves de contrôle : les formulaires de mathématiques côtoyaient, plus intéressant, les dictionnaires de langue allemande pour les épreuves d'allemand, ainsi que les dictionnaires unilingues pour les épreuves de langues étrangères.

Même si nous avons dit (cf. note $\mathrm{n}^{\circ} 1$ ) que nous n'aborderions pas ici la question du temps et de la journée scolaire en Allemagne, il est évident que l'attention portée à l'appropriation personnelle de ses outils par l'élève est d'autant plus développée que l'élève travaille souvent chez lui, seul, et pour des demi-journées entières.

\section{DES DÉMARCHES PÉdAgOgIQUES CONSTAMMENT EXPLICITÉES}

Le plus étonnant pour celui qui procède ainsi à cette plongée rapide dans un univers scolaire allemand tient sans doute au fait que les élèves avaient visiblement des choses à dire sur leur propre travail, et qu'ils avaient envie de les dire. Cette disponibilité à traiter d'une question pédagogique importante venait certes pour partie de ce que leur propre itinéraire les avait souvent mis en position de comparer des systèmes éducatifs, caractéristique qui serait alors propre à ces lycéens d'une école allemande " expatriée ».

La question en fait ne se réduit pas là :

- le fait d'avoir à arrêter avec leurs professeurs des points du programme qui seront l'objet des approfondissements de l'année joue sans doute un rôle positif dans l'apprentissage-même, non seulement parce qu'il responsabilise les parties, mais aussi parce qu'il oblige à repenser constamment le rapport entre le savoir scolaire et la finalité de l'apprentissage ; des questions qui sont en général du ressort du seul professeur ${ }^{8}$ deviennent en ce cas de la compétence partagée de l'élève ;

- à l'intérieur - même de la classe l'inexistence proclamée d'un point de vue «savant» se présentant d'abord comme tel peut laisser sceptique, voire inquiéter, et il faudrait faire appel à des travaux de recherche pour passer d'une opinion exprimée à une connaissance objective de la figure de l'enseignant allemand comparée à celle, par exemple, de son homologue français : ce qui est en tout état de cause frappant c'est la conscience très vive qu'avaient les élèves et le consensus qui paraissait régner dans l'établissement, autour de cette idée que

8. L'usage spontané et maîtrisé par les élèves eux-mêmes de mots du vocabulaire pédagogique pour décrire leurs propres travaux est surprenant : citons entre autres « reproduction », « transfert », « jugement personnel ». 
le professeur était celui qui entraînait ses élèves en travaillant avec eux plutôt que celui qui dispensait un savoir.

N'appelant pas de conclusion, cet article donne leur prix aux deux questions suivantes :

Si le programme d'enseignement peut parfois, dans certains systèmes éducatifs, être perçu comme un carcan qui contraint à l'excès les activités de la classe, ne peut-on imaginer des modalités de gestion de ces programmes ${ }^{9}$ qui évitent mieux que d'autres cette contradiction?

Si l'on sait que partout les épreuves des examens ont un rôle important de modélisation en amont des activités proposées aux élèves, ne faut-il pas s'assurer que les systèmes d'épreuves en vigueur ne démentent pas beaucoup des efforts réalisés par ailleurs pour que l'élève soit actif, et de plus en plus autonome, dans son travail?

9. Est-il intéressant de noter qu'au cours des entretiens il n'a été fait nulle mention de quelque « interdisciplinarité » à rechercher particulièrement, ni d'un changement que les technologies contemporaines auraient apporté au travail des élèves? 